\title{
SOME REMARKS ON $q$-BETA INTEGRAL
}

\author{
W. A. AL-SALAM AND A. VERMA
}

ABSTRACT. The following $q$-integral

$$
\int_{-c}^{d} \frac{(-q t / c)_{\alpha-1}(q t / d)_{\beta-1}}{(-q e t)_{\alpha+\beta}} d_{q} t
$$

is evaluated. A more general $q$-integral is also considered. Some applications to the $q$-Wilson (or Askey-Wilson) polynomials are also given.

1. Introduction. In a recent paper [1] Andrews and Askey gave the $q$-beta integral

$$
\begin{aligned}
\int_{-c}^{d} \frac{(-q x / c ; q)_{\infty}(q x / d ; q)_{\infty}}{\left(-q^{\alpha} x / c ; q\right)_{\infty}\left(q^{\beta} x / d ; q\right)_{\infty}} d_{q} x \\
=\frac{c d(1-q)(q ; q)_{\infty}\left(q^{\alpha+\beta} ; q\right)_{\infty}(-c / d ; q)_{\infty}(-d / c ; q)_{\infty}}{(c+d)\left(q^{\alpha} ; q\right)_{\infty}\left(q^{\beta} ; q\right)_{\infty}\left(-q^{\beta} c / d ; q\right)_{\infty}\left(-q^{\alpha} d / c ; q\right)_{\infty}} \\
=\frac{\Gamma_{q}(\alpha) \Gamma_{q}(\beta)}{\Gamma_{q}(\alpha+\beta)} \frac{c d}{c+d} \frac{(-c / d ; q)_{\infty}(-d / c ; q)_{\infty}}{\left(-q^{\beta} c / d ; q\right)_{\infty}\left(-q^{\alpha} d / c ; q\right)_{\infty}}
\end{aligned}
$$

The last equality holds for $0<q<1$. For notation we refer the reader to [1]. However, since base $q$ is not changed throughout this work we shall write $(a)_{n}$ for $(a ; q)_{n}$. If, in addition, we write $(z)_{\alpha}=(z)_{\infty} /\left(z q^{\alpha}\right)_{\infty}$ then $(1.1)$ can be written more compactly (and perhaps more suggestively) as

$$
\int_{-c}^{d}(-q x / c)_{\alpha-1}(q x / d)_{\beta-1} d_{q} t=\frac{\Gamma_{q}(\alpha) \Gamma_{q}(\beta)}{\Gamma_{q}(\alpha+\beta)} \frac{c d}{c+d}\left(-\frac{c}{d}\right)_{\beta}\left(-\frac{d}{c}\right)_{\alpha} .
$$

In this note we first generalize (1.2) to obtain the $q$-beta formula

$$
\int_{-c}^{d} \frac{(-q t / c)_{\alpha-1}(q t / d)_{\beta-1}}{(-q e t)_{\alpha+\beta}} d_{q} t=\frac{c d}{c+d} \frac{\Gamma_{q}(\alpha) \Gamma_{q}(\beta)}{\Gamma_{q}(\alpha+\beta)} \frac{(-d / c)_{\alpha}(-c / d)_{\beta}}{(-q e d)_{\alpha}(-q e c)_{\beta}}
$$

which is a $q$-analog of a formula of Dinghas [4, Formula (2.4)] and which reduces to the Andrews-Askey formula (1.1) when $e=0$. We shall obtain this formula first by using the transformation formula of Sears [4, (5.2)] and then by another method.

We next consider the still more general integral

$$
I=\int_{-c}^{d} \frac{(-q t / c)_{\alpha-1}(q t / d)_{\beta-1}}{(-q e t / c)_{\gamma}(q f t / d)_{\delta}} d_{q} t
$$

for $\alpha+\beta=\gamma+\delta$. We show that $I$ can be written as a well-poised ${ }_{8} \Phi_{7}$ (see formula (3.1) below).

Finally we use (1.4) to give $q$-integral representation for the general $q$-Wilson [2] ${ }_{4} \Phi_{3}$ polynomials.

Received by the editors July 16,1981 .

1980 Mathematics Subject Classification. Primary 33A15, 33A65.

Key words and phrases. $q$-beta integrals, Askey-Wilson polynomials, basic series.

(c) 1982 American Mathematical Society $0002-9939 / 81 / 0000-0357 / \$ 01.75$ 
2. Formula (1.3). Sears proved the identity [5, (5.2)]

$$
\begin{aligned}
\frac{(e)_{\infty}(f)_{\infty}}{(a)_{\infty}(b)_{\infty}(c)_{\infty}}{ }_{3} \Phi_{2}\left[\begin{array}{c}
a, b, c ; q \\
e, f
\end{array}\right]-\frac{q}{e} \frac{\left(q^{2} / e\right)_{\infty}(q f / e)_{\infty}}{(q a / e)_{\infty}(q b / e)_{\infty}(q c / e)_{\infty}} \\
\cdot{ }_{3} \Phi_{2}\left[\begin{array}{c}
q a / e, q b / e, q c / e ; q \\
q^{2} / e, q f / e
\end{array}\right]=\frac{(e)_{\infty}(q / e)_{\infty}(f / a)_{\infty}(f / b)_{\infty}(f / c)_{\infty}}{(a)_{\infty}(b)_{\infty}(c)_{\infty}(q a / e)_{\infty}(q b / e)_{\infty}(q c / e)_{\infty}}
\end{aligned}
$$

where $a b c q=e f$.

If in this formula we put $a=-d q^{\alpha} / c, b=q^{\beta}, c=-e d q, e=-d q / c$ and $f=-e d q^{\alpha+\beta+1}$ we get, after some manipulation, formula (1.3).

3. The integral $I$. Let $\alpha+\beta=\gamma+\delta$ and consider the $q$-integral $I$ in (1.4). By definition we can write

$$
\begin{aligned}
I= & \frac{(-d q / c)_{\alpha-1}(q)_{\beta-1} d(1-q)}{(f q)_{\delta}(-d e q / c)_{\gamma}}{ }_{4} \Phi_{3}\left[\begin{array}{c}
-q^{\alpha} d / c, q^{\beta},-e d q / c, f q ; q \\
f q^{1+\delta},-d q / c,-d e q^{1+\gamma} / c
\end{array}\right] \\
& +c(1-q) \frac{(q)_{\alpha-1}(-c q / d)_{\beta-1}}{(e q)_{\gamma}(-c f q / d)_{\delta}}{ }_{4} \Phi_{3}\left[\begin{array}{c}
q^{\alpha},-c f q / d,-c q^{\beta} / d, e q ; q \\
e q^{1+\gamma},-c q / d,-c f q^{1+\delta} / d
\end{array}\right]
\end{aligned}
$$

Now making use of the $q$-analog of Whipple's theorem [3, p. 69], which expresses a well-poised ${ }_{8} \Phi_{7}$ as a combination of two Saalschützian ${ }_{4} \Phi_{3}$, we get

$$
\begin{aligned}
I= & (c+d)(1-q)(q)_{\infty}(-c q / d)_{\beta-1}(-d q / c)_{\alpha-1} /(e q)_{\gamma-\alpha} \\
& \cdot{ }_{8} \Phi_{7}\left[\begin{array}{c}
A, q \sqrt{A},-q \sqrt{A}, q^{\beta}, f q,-e d q / c,-f c q^{\delta-\alpha+1} / d, e q^{\gamma-\alpha+1} ; q^{\alpha} \\
\sqrt{A},-\sqrt{A}, e f q^{2}, e q^{1+\beta},-f c q^{1+\beta} / d,-e d q^{\alpha+\beta-\delta+1} / c, f q^{\alpha-\gamma+1}
\end{array}\right]
\end{aligned}
$$

where $A=e f q^{1+\beta}$.

Formula (2.1) also implies (1.3) by taking $f=\delta=0$ and using the formula

$$
{ }_{2} \Phi_{1}[a, b ; c ; c / a b]=\frac{(c / a)_{\infty}(c / b)_{\infty}}{(c)_{\infty}(a / a b)_{\infty}} .
$$

Returning to (3.1), we now recall a transformation of Bailey [3, p. 70]

$$
\begin{aligned}
{ }_{8} \Phi_{7}\left[\begin{array}{c}
A, q \sqrt{A},-q \sqrt{A}, B, C, D, E, F ; A^{2} q^{2} / B D E F \\
\sqrt{A},-\sqrt{A}, q A / B, q A / C, q A / D, q A / E, q A / F
\end{array}\right] \\
\quad=\frac{(A q)_{\infty}(A q / D E)_{\infty}(A q / D F)_{\infty}(A q / E F)_{\infty}}{(A q / D)_{\infty}(A q / E)_{\infty}(A q / F)_{\infty}(A q / D E F)_{\infty}} \Phi_{3}\left[\begin{array}{c}
A q / B C, D, E, F ; q \\
D E F / A, A q / B, A q / C
\end{array}\right]
\end{aligned}
$$

where the series on the RHS terminates whereas the series on the L.H.S. may not terminate.

With the aid of this transformation we see that

$$
\begin{aligned}
I= & (c+d)(1-q) \frac{(q)_{\infty}(-c q / d)_{\beta-1}(-d q / c)_{\alpha-1}}{(e q)_{\gamma-\alpha}} \\
& \cdot \frac{(-f c q / d)_{\beta}\left(-e d q^{1+\alpha-\delta} / c\right)_{\beta}}{\left(e f q^{2}\right)_{\beta}\left(q^{\alpha-\delta}\right)_{\beta}} \cdot{ }_{4} \Phi_{3}\left[\begin{array}{c}
q^{\delta}, q^{\beta},-e d q / c,-f c q^{\delta-\alpha+1} / d ; q \\
q^{1+\delta-\alpha}, e q^{1+\beta}, f q^{1+\delta}
\end{array}\right]
\end{aligned}
$$


provided that either $q^{\delta}, q^{\beta},-e d q / c$, or $-f c q^{\delta-\alpha+1} / d$ is of the form $q^{-n}$ where $n=0,1,2,3, \ldots$.

This formula suggests the following representation for the $q$-Wilson polynomial

$$
W_{n}(x)={ }_{4} \Phi_{3}\left[\begin{array}{c}
q^{-n}, a b q^{1+n}, q^{-x}, c d q^{1+x} ; q \\
a q, c q, b d q
\end{array}\right]
$$

namely,

$$
\begin{gathered}
W_{n}(x)=\frac{\left(a q^{1+x}\right)_{\infty}\left(c d q^{1+x}\right)_{\infty}\left(q^{-x}\right)_{\infty}\left(b q^{-x} / c\right)_{\infty}\left(a q^{-x} / c d\right)_{\infty}\left(b d q^{1+x}\right)_{\infty}}{(1-q)(q)_{\infty}(a q)_{\infty}\left(d q^{1+x}-q^{-x} / c\right)\left(q^{-n} / c\right)_{n}\left(q^{-2 x} / c d\right)_{\infty}\left(c d q^{2+2 x}\right)_{\infty}} \\
\cdot \frac{1}{(b d q)_{\infty}\left(a b q^{1+n} / c\right)_{\infty}} \int_{c^{-1} q^{-x}}^{d q^{1+x}} \frac{\left(a b t q^{1+n}\right)_{\infty}\left(t q^{-n}\right)_{\infty}\left(c t q^{1+x}\right)_{\infty}\left(t q^{-x} / d\right)_{\infty}}{(b t)_{\infty}(a t / d)_{\infty}(c t)_{\infty}(t)_{\infty}} d_{q} t
\end{gathered}
$$

or the representation,

$$
\begin{aligned}
W_{n}(x)= & \frac{\left(q^{-n} / b\right)_{\infty}\left(d^{2} q^{2+x}\right)_{\infty}(1 / c)_{\infty}\left(a q^{1+x}\right)_{\infty}\left(a b q^{1+n}\right)_{\infty}\left(b q^{-x} / c\right)_{\infty}\left(q^{-x}\right)_{\infty}}{(1-q)(q)_{\infty}(a q)_{\infty}\left(b q^{n-x}\right)_{\infty}(b d q)_{\infty}\left(q^{x+1-n} / b\right)_{\infty}} \\
& \cdot \frac{q^{-x}}{\left(q^{-x} / c\right)_{\infty}\left(a b q^{1+n} / c\right)_{\infty}} \\
& \cdot \int_{b q^{n}}^{q^{x}} \frac{\left(t q^{1-x}\right)_{\infty}\left(t q^{1-n} / b\right)_{\infty}\left(a t q^{1-x} / c\right)_{\infty}\left(d t q^{1-n}\right)_{\infty}}{(t d q)_{\infty}\left(t q^{-x-n} / b\right)_{\infty}(t q a)_{\infty}\left(t q^{-x-n} / c\right)_{\infty}} d_{q} t .
\end{aligned}
$$

The latter implies the representation for the $q$-Meixner polynomial

$$
\begin{aligned}
{ }_{2} \Phi_{1}\left[\begin{array}{c}
q^{-n}, q^{-x} ; b q^{1+n} \\
c q
\end{array}\right]= & \frac{q^{-x}\left(q^{-x}\right)_{\infty}\left(b q^{-x} / c\right)_{\infty}(1 / c)_{\infty}\left(q^{-n} / b\right)_{\infty}}{(1-q)(q)_{\infty}\left(q^{-x} / c\right)_{\infty}\left(b q^{n-x}\right)_{\infty}\left(q^{1+x-n} / b\right)_{\infty}} \\
& \cdot \int_{b q^{n}}^{q^{x}} \frac{\left(t q^{1-x}\right)_{\infty}\left(t q^{1-n} / b\right)_{\infty}}{\left(t q^{-x-n} / b\right)_{\infty}\left(t q^{-x-n} / c\right)_{\infty}} d_{q} t .
\end{aligned}
$$

\section{REFERENCES}

1. G. E. Andrews and R. Askey, Another q-extension of the beta function, Proc. Amer. Math. Soc. 81 (1981), 97-100.

2. R. Askey and J. Wilson, A set of orthogonal polynomials that generalize the Racah coefficients of 6 - j symbols, Mathematics Research Center, University of Wisconsin-Madison, MRC Technical Summary Report \#1833.

3. W. N. Bailey, Generalized hypergeometric series, Cambridge Univ. Press, New York, 1935.

4. A. Dinghas, Zur Darstellung einger Klassen hypergeometrischer Polynome durch Integrale vom Dirichlet-Mehlerschen Typus, Math. Z. 53 (1950), 76-83.

5. D. B. Sears, Transformation of basic hypergeometric functions of special type, Proc. London Math. Soc. 52 (1951), 467-483.

Department of MAThematics, University of Alberta, Edmonton, Alberta, CANADA T6G 2G1

DEPARTMENT OF MATHEMATICS, ROORKEE UNIVERSITY, ROORKEE, INDIA 